\title{
Spatially and Temporally Resolved Measurements of Turbulent Rayleigh-Bénard Convection by Lagrangian Particle Tracking of Long-Lived Helium-Filled Soap Bubbles
}

\author{
J. Bosbach*, D. Schanz, P. Godbersen, A. Schröder \\ Department of Experimental Methods, Institute of Aerodynamics and Flow Technology, \\ German Aerospace Center (DLR), Göttingen, Germany \\ * Johannes.Bosbach@dlr.de
}

\begin{abstract}
We present spatially and temporally resolved velocity and acceleration measurements of turbulent RayleighBénard convection spanning the whole volume $\left(\sim 1 \mathrm{~m}^{3}\right)$ of a cylindrical sample with aspect ratio one. With the "Shake-The-Box" (STB) Lagrangian particle tracking (LPT) algorithm, we were able to instantaneously track up to 560,000 particles, corresponding to mean inter-particle distances down to $6-8$ Kolmogorov lengths. We used the data assimilation scheme 'FlowFit', which involves continuity and Navier-Stokesconstraints, to map the scattered velocity and acceleration data on cubic grids, herewith recovering the smallest flow scales. Lagrangian and Eulerian visualizations reveal the dynamics of the large-scale circulation and its interplay with small scale structures, such as thermal plumes and turbulent background fluctuations. As a result, the complex time-dependent behavior of the LSC comprising azimuthal rotations, torsional oscillation and sloshing can be extracted from the data. Further, we found more seldom dynamic events, such as spontaneous reorientations of the LSC in the data from long-term measurements.
\end{abstract}

\section{Introduction}

Rayleigh-Bénard convection (RBC), where a fluid is heated from below and cooled from above under welldefined conditions, is the most generic type of thermal convection and a configuration suited ideally to study the fundamentals of buoyancy driven flows (Bodenschatz et al. 2000; Chillà and Schumacher 2012). It is characterized by three dimensionless parameters, which are the aspect ratio $\Gamma$, the Prandtl number Pr and the Rayleigh number Ra. Herewith, the aspect ratio is defined by $\Gamma=\mathrm{D} / \mathrm{L}, \mathrm{D}$ being the diameter and $\mathrm{L}$ the height of the fluid sample while the Prandtl number $\operatorname{Pr}=v / \kappa$ is a fluid property and given by the ratio of kinematic viscosity $v$ and thermal diffusivity $\kappa$. The actual driving of the flow due to the applied temperature gradient is quantified by the Rayleigh number, which is a dimensionless measure for the ratio of buoyancy and dissipative forces and given by $\mathrm{Ra}=\beta \mathrm{gL}^{3} \Delta \mathrm{T} /(\mathrm{v}), \beta$ and $\mathrm{g}$ being the isobaric thermal expansion coefficient and the acceleration of gravity, respectively.

In many fluids of practical relevance, even for moderate temperature gradients, Ra is already above a critical value such that the convective flow between the plates is turbulent. A prominent feature of the turbulent RB system is the presence of a large-scale circulation (LSC). It develops from self-organization of the thermal plumes, that erupt from the top and bottom thermal boundary layers. In cylindrical fluid samples of aspect ratios close to one with a high degree of symmetry, the LSC reveals complex short- and long-term dynamics 
involving rotations, cessations and restarts (Brown and Ahlers 2006a), torsional oscillations (Funfschilling et al. 2008; Zhou et al. 2009) and sloshing modes (Zhou et al. 2009). Even the earths Coriolis force was found to influence the long-term motion of the LSC (Brown and Ahlers 2006b).

Although the dynamics of the LSC in turbulent RBC has gained much attention in the past, direct volumetric measurements of the LSC are still rare. While tomographic PIV (Paolillo et al. 2018; Schiepel et al. 2018) or LPT (Liot et al. 2017; Schiepel et al. 2018) measurements have been reported recently, measurements at full spatial and temporal resolution covering the whole sample were still lacking. The same holds for direct measurements of the velocity fields of the LSC and its Lagrangian transport properties. To bridge this gap, we performed the experiments and analyses described in the following.

\section{Experimental Set-Up}

\subsection{RBC Apparatus}

We developed a dedicated convection experiment, which allowed generating classical turbulent RBC and at the same time application of time-resolved LPT in the whole fluid volume (Figure 1). The convection cell has an aspect ratio of $\Gamma=1$ and a height of $\mathrm{L}=1.1 \mathrm{~m}$. By using air at atmospheric pressure as working fluid $(\operatorname{Pr} \sim 0.7)$, Rayleigh numbers up to $R a \sim 10^{9}$ could be reached with moderate temperature differences.
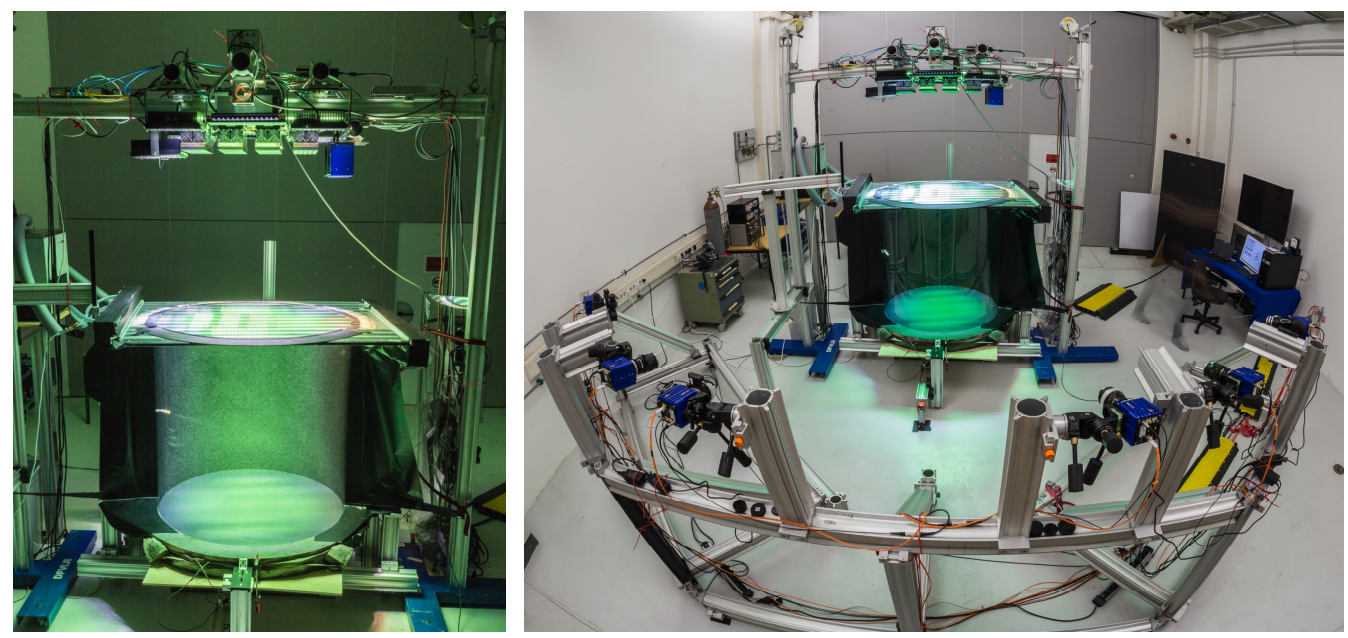

Figure 1: Convection cell (left) and optical set-up with six scientific CMOS cameras and array of pulsed LEDs for time-resolved LPT with long-lived HFSB (right) (photographic work by J. Agocs)

Thermal control of the experiment is enabled by an electrically heated aluminum plate with a thickness of $15 \mathrm{~mm}$ and a water-perfused cooling plate, which allows for illumination of the sample from the top. For the sake of a high particle image quality, the sidewall consisted of two bended sheets of acrylic glass with a thickness of $2 \mathrm{~mm}$ only. The heating plate and the rear part of the sidewall were covered with a self-adhesive, matte black foil to reduce optical reflections and stray light from the light source to a minimum.

For the temperature measurements, a dedicated temperature logger based on an array of integrated ICs with constant current source, 24-bit ADC and I2C interface was built for this purpose and operated in combination with 1/3 DIN B Pt1000 resistance temperature detectors (RTDs), connected in four-wire mode. The sensors were placed at three positions in the heating plate, at the in- and outflow of the cooling plate and at two positions in the air surrounding the cell.

\subsection{Long-Lived Helium-Filled Soap Bubbles}

Due to the large dimensions of the experiment, tracer particles suitable for air as working fluid with high scattering efficiency are required. For this purpose, sub millimeter helium filled soap bubbles have been 
established as the tracer of choice for mixed convective flows (Bosbach et al. 2009; Kühn et al. 2009, 2012) and recently for low speed wind tunnel (Gibeau et al. 2020; Schanz et al. 2019; Scarano et al. 2015) and even free field tests (Spoelstra et al. 2019). However, their application to RBC was impeded by the limited lifetime before. Hence, specifically for investigation of large-scale thermal convection, we developed a bubble film solution (BFS), which provides a much longer bubble lifetime as compared to commercial fluids and thus allows for measurement runs as long as 30 minutes. Herewith, the main constraint was given by the necessity that we wanted the nozzle design to remain unchanged, which limited the amount and candidates of polymers, which could be used in the BFS. The final solution is composed besides water and additives of minor impact by a mixture of several surfactants and polymers. Herewith, the challenge was to provide as stable as possible operation while maintaining the required shell thickness for neutral buoyancy conditions $(\sim 60 \mathrm{~nm})$. The HFSB were generated with the orifice nozzle of DLR (Bosbach et al. 2009) in combination with a commercial HFSB controller (LaVision). The resulting HFSB have a mean diameter of $370 \mu \mathrm{m}$. However, while we tried as good as possible to maintain a continuous generation by the nozzle, the operation retained slightly intermittent with productive phases (see Figure 2 (left)) and null phases changing at a rate of the order of $1 \mathrm{~Hz}$.

As an added benefit the HFSB residence time in the sample can be evaluated from the LPT data, see section 4. Hereto, we evaluated the number of tracked particles for a long-term run in Figure 2. An exponential function is fitted to the data (dotted line), revealing an exponential decay of $\mathrm{N}(\mathrm{t})$ after an initial rise during the first 110 time-steps to a maximum of 560,000 particles. The time constant of the exponential fit indicates a mean HFSB residence time in the RBC experiment of $\tau_{\mathrm{r}}=(326 \pm 5) \mathrm{s}$. For a commercial BFS on the other hand, a mean lifetime of $97 \mathrm{~s}$ has been measured before (Huhn et al. 2017), demonstrating, that the new BFS allows for a tremendous extension of the HFSB life time.
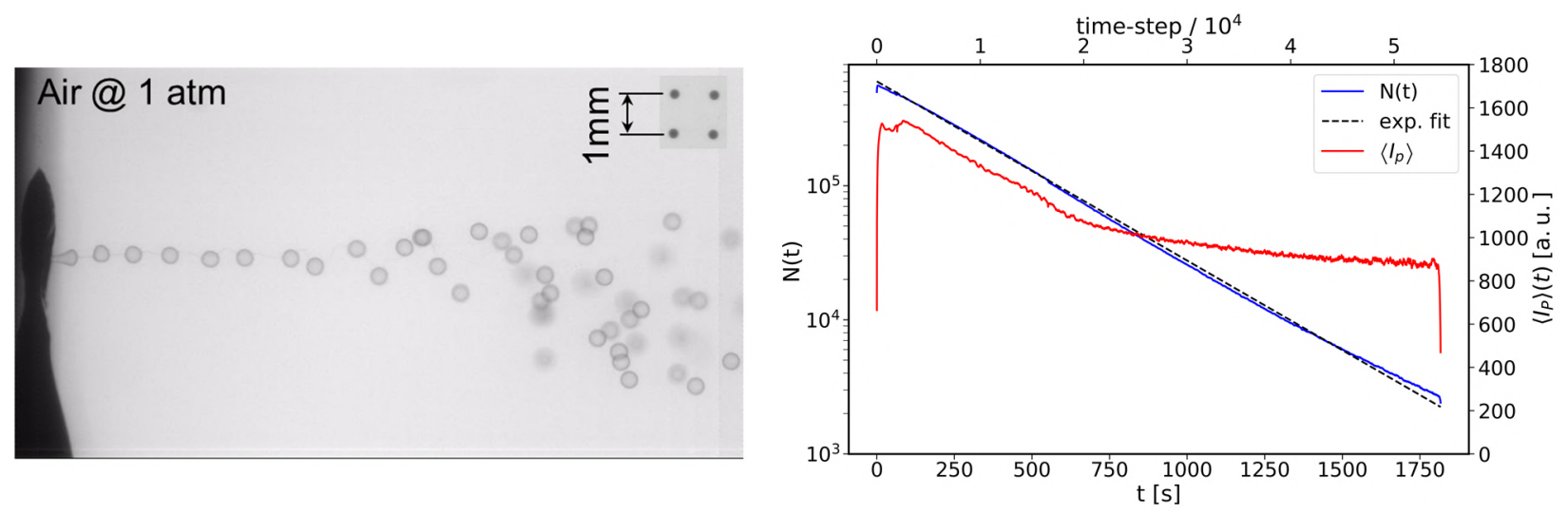

Figure 2: Shadowgraph of HFSB generation (productive phase) (left). Number of tracked particles N(t) and intensity of reconstructed particles $\mathrm{I}_{\mathrm{p}}(\mathrm{t})$ for run 30 , evaluated at every 10th time-step (right). The dotted line is a fit of an exponential function with a time-constant of $\tau_{\mathrm{r}}=326 \mathrm{~s}$.

The LPT data further revealed, that the residence time of the particles is not only influenced by the limited lifetime but also by a settling velocity of $\sim 1.4 \mathrm{~mm} / \mathrm{s}$, which had to be accepted as a tradeoff between lifetime and achievable shell thickness. However, considering the measured settling velocity, we estimated the Stokes number of the HFSB to amount

$$
S t=\frac{\tau_{p}}{\tau_{\eta}}=(8.0 \pm 1.7) \cdot 10^{-4} .
$$

Herewith, $\tau_{\mathrm{p}}$ denotes the well-known particle response time and $\tau_{\eta}$ the mean Kolmogorov time of the flow. As $S t<10^{-3}$, the HFSB should be able to follow all turbulent fluctuations in our RBC experiment.

Looking at the intensity of the reconstructed particles given in Figure 2, a decrease of the mean brightness by about $50 \%$ during the first ten minutes of the experiment can be detected. As the measurement continues, the intensity stays rather constant. We ascribe this effect to the secondary scattering of the HFSB at the 
initially high seeding densities, which diminishes as soon as the seeding density drops below about 0.1 HFSB per cubic centimeter.

\subsection{Optical Set-Up}

Illumination of the tracer particles in the sample was enabled by an installation of 849 pulsed high-power LEDs above the transparent cooling plate. Hereto, different arrays of LED spots in either white or green color were used. By lifting the LED array to a level of about $\sim 1 \mathrm{~m}$ above the cooling plate we ensured that the gaps between the LED modules were sufficiently filled with light in the sample volume. The LEDs were synchronized with the camera system and operated in pulsed mode with a pulse duration of $3 \mathrm{~ms}$. To capture the images of the illuminated HFSBs, we used an inline ensemble of six scientific CMOS cameras with a resolution of $2560 \times 2159$ pixels each, which we combined with Zeiss Distagon lenses ( $\mathrm{f}=35 \mathrm{~mm}$ ). They were installed on a circle around the convection cell at a distance of about $3 \mathrm{~m}$ from the vertical center line and covered an aperture of $69^{\circ}$. Sharp imaging of the particles was achieved by closing the apertures to $\mathrm{F} / 9.5$ and $\mathrm{F} / 11$. The mean magnification in the volume amounted to $\mathrm{M}=0.012$, corresponding to a resolution of $0.55 \mathrm{~mm} / \mathrm{px}$. Accordingly, the geometric particle images would measure about 0.7 pixels in diameter. In contrast, the real particle image diameters ranged between 2 and 3 pixels due to diffraction limited imaging. These values allow for precise sub-pixel fitting, yet are not suited to resolve the glare points of the HFSBs. Calibration of the camera system was done using a quadratically arranged dot pattern (spacing $25 \mathrm{~mm}$ ) on an aluminum honeycomb plate, which we placed in three parallel planes with a consecutive distance of $230 \mathrm{~mm}$ within the cell. This geometrical calibration was further refined using Volume Self Calibration (Wieneke 2008) and calculation of the optical transfer function (Schanz et al. 2013) using low density images from dedicated runs. Both, image acquisition and synchronization of the cameras and the light source were controlled through the DaVis 10.0.5 software (LaVision).

\section{Experimental Conditions and Measurement Procedure}

Preceding each measurement, the convection apparatus was given between three and four hours for thermal equilibration of the respective boundary conditions. Herewith, the temperature levels of heating $\left(\mathrm{T}_{\mathrm{h}}\right)$ and cooling plate $\left(T_{c}\right)$ were chosen such that the mean sample temperature $\langle T\rangle=\left(T_{h}+T_{c}\right) / 2$ equaled the mean room temperature in the surrounding of the experiment. Prior to the LPT measurements, we seeded the fluid with HFSBs by temporally inserting the HFSB nozzle through a small opening in the side wall for up to 4 minutes. In order to prevent induction of long-lasting flow structures, we changed the direction of the HFSB nozzle every $10 \mathrm{~s}$. After seeding, the system was given between two and four minutes to allow for the turbulence induced in excess by the HFSB nozzle to decay. We conducted continuous and chunked (a multitude of short recordings with limited time-steps to attain a long overall runtime or statistical convergence) measurement runs with durations between $80 \mathrm{~s}$ and 30 minutes. In total, 30 experimental runs of varying duration and interval configuration were performed. While the heat impact by the lights sources was negligible during the short or chunked runs, the central heating element was switched off during the long-term measurements. Hereto, the optical power of the LEDs was adjusted in order to exactly compensate for the heat flux of the central heating element.

From the complete data of the measurement campaign, we selected two for this study in order to discuss the measurement procedure and the quality of the resulting data. The boundary conditions and measurement parameters for these runs, which comprise two Ra, are denoted in Table 1 and 2. As an indication of the range of length- and time-scales to be expected, the volume-averaged Kolmogorov length- and time-scales are given (Sugiyama et al. 2007). Further, the nominal thickness of the thermal boundary layer, from which the thermal plumes are emitted, is calculated via the relation $\lambda_{b}=1 /(2 N u)$ (Chillà and Schumacher 2012)

and the "free-fall" velocity $U_{f}=\sqrt{\beta g \Delta T L}$ is added as a measure for the maximal velocities to expect (Chillà and Schumacher 2012). Given the initial density of tracked particles between 0.32 and $0.56 / \mathrm{cm}^{3}$, the measurements begin with mean particle distances between 6.4 and 7.8 Kolmogorov lengths. As the lifetime of 
the tracer particles was limited, the seeding density further decayed during the experimental runs. Accordingly, data assimilation by FlowFit is required in order to resolve the smallest spatial scales in the Eulerian frame. The temporal oversampling, in turn, corresponds to 5.4 and 7.0 mean Kolmogorov time constants, allowing to resolve the smallest temporal scales and the spatial scales along the Lagrangian tracks. While the short experimental 'run 09' of 3384 time-steps has a duration of about 60 free fall times, the long-term 'run 30' spans more than 1,000 free fall times. According to the results in Table 1, the smallest turbulent scales in the flow are already 4-5 times larger than the tracer particles and should be fully resolvable by the measurement system. The same holds for the thermal boundary layers, which measure more than $\sim 20$ nominal particle diameters.

Table 1: Boundary conditions and fundamental parameters of the presented flow cases. $\langle T\rangle, \eta_{K}, \tau_{\eta}, \lambda_{b}$ and $\mathrm{U}_{\mathrm{f}}$ denote the mean sample temperature, Kolmogorov length and time-scale, the thermal boundary layer thickness and free-fall velocity, respectively. For $\Delta \mathrm{T},\langle\mathrm{T}\rangle$ and $\mathrm{Ra}$, the maximal deviations occurring during the STB measurement are denoted.

\begin{tabular}{|c|c|c|c|c|c|c|c|c|}
\hline run \# & $\Delta \mathrm{T}[\mathrm{K}]$ & $\langle\mathrm{T}\rangle\left[{ }^{\circ} \mathrm{C}\right]$ & $\operatorname{Ra}\left[10^{8}\right]$ & $\operatorname{Pr}$ & $\eta_{\mathrm{K}}[\mathrm{mm}]$ & $\tau_{\eta}[\mathrm{s}]$ & $\lambda_{\mathrm{b}}[\mathrm{mm}]$ & $\mathrm{U}_{\mathrm{f}}[\mathrm{m} / \mathrm{s}]$ \\
\hline 09 & $4.03 \pm 0.06$ & $22.00 \pm 0.06$ & $5.25 \pm 0.06$ & 0.70 & 2.3 & 0.35 & 11.5 & 0.38 \\
\hline 30 & $11.8 \pm 0.2$ & $22.14 \pm 0.17$ & $15.3 \pm 0.3$ & 0.70 & 1.7 & 0.18 & 8.5 & 0.66 \\
\hline
\end{tabular}

Table 2: Experimental settings and measurement parameters for the flow cases given in Table 1. Herewith, $\mathrm{f}_{\mathrm{acq}}, \mathrm{D}$ and $\mathrm{N}$ denote the acquisition frequency, the duty cycle of the LEDs and number of instantaneous recordings, respectively. $\mathrm{N}_{\mathrm{f}}$ denotes the acquisition time in number of free fall times. Finally, the maximal number of tracked particles $\mathrm{n}$, the mean interparticle distance $\Delta / \eta_{\mathrm{K}}$, and the temporal oversampling $\tau_{\eta} \cdot \mathrm{f}$ are specified.

\begin{tabular}{|c|c|c|c|c|c|c|c|}
\hline run \# & $\mathrm{f}_{\text {acq }}[\mathrm{Hz}]$ & $\mathrm{D}$ & $\mathrm{N}$ & $\mathrm{N}_{\mathrm{f}}$ & $\mathrm{n}\left[1 / \mathrm{cm}^{3}\right]$ & $\Delta / \eta_{\mathrm{K}}$ & $\tau_{\eta} \cdot \mathrm{f}$ \\
\hline 09 & 20 & 0.15 & 3384 & 59 & 0.32 & 6.4 & 7.0 \\
\hline 30 & 30 & 0.1 & 54528 & 1091 & 0.56 & 7.8 & 5.4 \\
\hline
\end{tabular}

\section{Data Processing}

The raw particle image data was processed using the "Shake-The-Box" LPT algorithm (Schanz et al. 2016) after slight image pre-processing, which consisted of subtraction of the sliding minimum of 36 consecutive images as well as a constant. The STB method employs a predictor/corrector approach, in which known tracks are first extended to the next time-step and subsequently treated by a position correction method ('shaking' with image matching techniques). This way, the vast majority of particles can be tracked effortlessly, avoiding ghost particle generation, before the small portion of yet untracked particles is identified using Iterative Particle Reconstruction IPR (Wieneke 2013) on the residual images. Images with locally up to 0.15 particles per pixel (ppp) were evaluated.

Due to the limited residence time of the HFSB, during the time-scale of the experiment, the number of tracks decays with time, see Figure 2. While after the second STB pass, more than half a million of HFSB are tracked at the first time-step, only a few thousand tracked HFSB remain after around 30 minutes. With the second STB pass, running backward in time and using the particle positions and track information from the first pass, the number of tracked particles can be increased by about $10 \%$. Hereafter, the particle tracks were fitted with a cubic B-spline curve using the 'TrackFit' algorithm (Gesemann et al. 2016), smoothing the particle tracks according to the found optimal filter length over about 5 time-steps. As a result, a particle position accuracy of $\sim 24 \mu \mathrm{m}(0.043 \mathrm{pixel})$ and a velocity uncertainty of $0.34 \mathrm{~mm} / \mathrm{s}$ ('run 30') could be estimated from the spatial spectrum of the tracks. The latter corresponds, depending on Ra, to a dynamic velocity range of $\sim 900: 1$ ('run30'). As the final step, the data assimilation scheme 'FlowFit' (Gesemann et al. 2016) with continuity and Navier-Stokes-constraints was used to interpolate the scattered velocity and 
acceleration data by continuous 3D B-Splines in a cubic grid at a grid spacing of $7 \mathrm{~mm}$, corresponding to 0.06 - 0.1 particles per B-spine cell (PPC).

As compared to earlier measurements with quite similar set-up and procedure (Huhn et al. 2017), much longer time-series were recorded and evaluated in this study and the number of tracked particles could be further increased by a factor of two.

\section{Results}

\subsection{Lagrangian Particle Tracks}
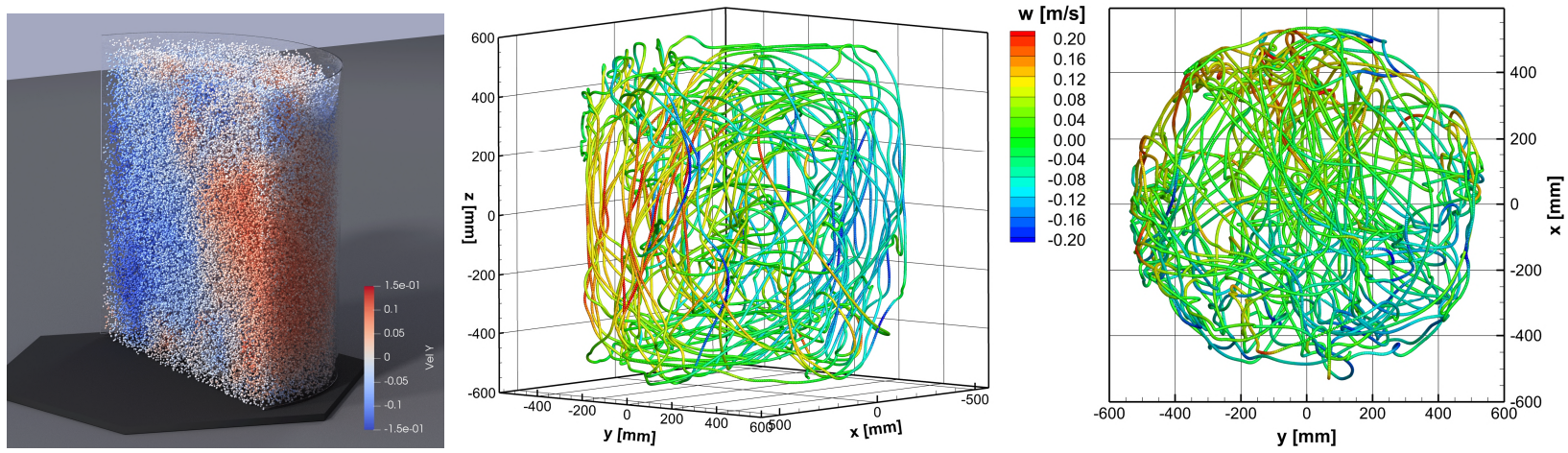

Figure 3: Volumetric particle distribution from 'run 09' (left), single particle trajectory of $\sim 4.4 \cdot 10^{4}$ timesteps from 'run 30' (middle, right). Vertical velocity component coded with color.
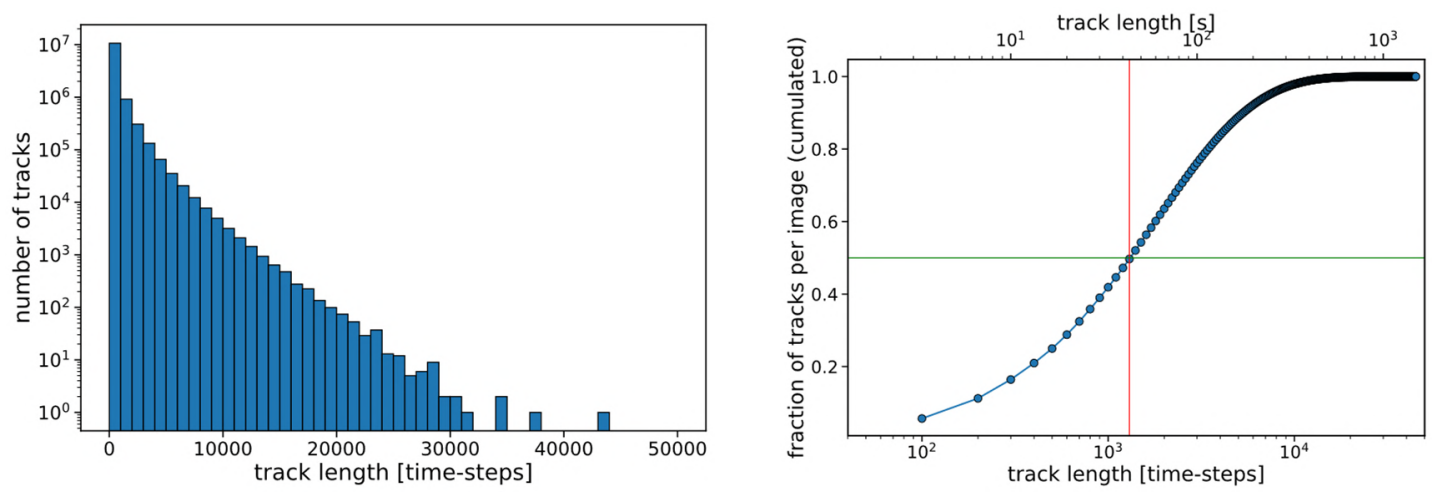

Figure 4: Distribution of track lengths for 'run 30'. Frequency distribution (left). Cumulated mean fraction of tracks per image (right). The green line indicates a value of 0.5 and the red line a track length of 1,300.

An exemplary output of the STB method is depicted in Figure 3 (left), which illustrates an instantaneous volumetric particle distribution of 'run 09' (Godbersen et al. 2020). For the sake of the moderate Ra, the LSC can be spotted well in the reconstructed distribution of $\sim 300,000$ tracer particles by looking at the color-coded vertical velocity. While the ability to perform long-time investigations is one of the assets of experimental studies of RBC, of course not all of the initially tracked HFSB could be observed over the full time-span of the measurement. The reasons for this are manifold. First, the HFSB have a limited residence time in the sample. However, this effect could only explain a minority of the track-endings. In fact, the majority of the tracks ends prior to reaching the end of residence of the according HFSB, which could be due to overlapping particle images, residual background reflections or high acceleration events. Accordingly, ending as well as newly beginning tracks are observed to some extent in all time-steps during the evaluation. However, the median length of the tracks per image totals to $\sim 1300$ time-steps $(\sim 43 \mathrm{~s})$. A few HFSB could be tracked over more than 30,000 and the longest trajectory found lasts 44,000 time-steps, 
which corresponds to about 900 free-fall times, 60 LSC turn-around times and a distance of 130 sample heights $(143 \mathrm{~m})$, see Figure 3. This opens up the possibility to perform long-time Lagrangian statistics with the larger fraction of the tracks over a significant number of time-steps.

\subsection{Global Flow Fields and Large-Scale Circulation}
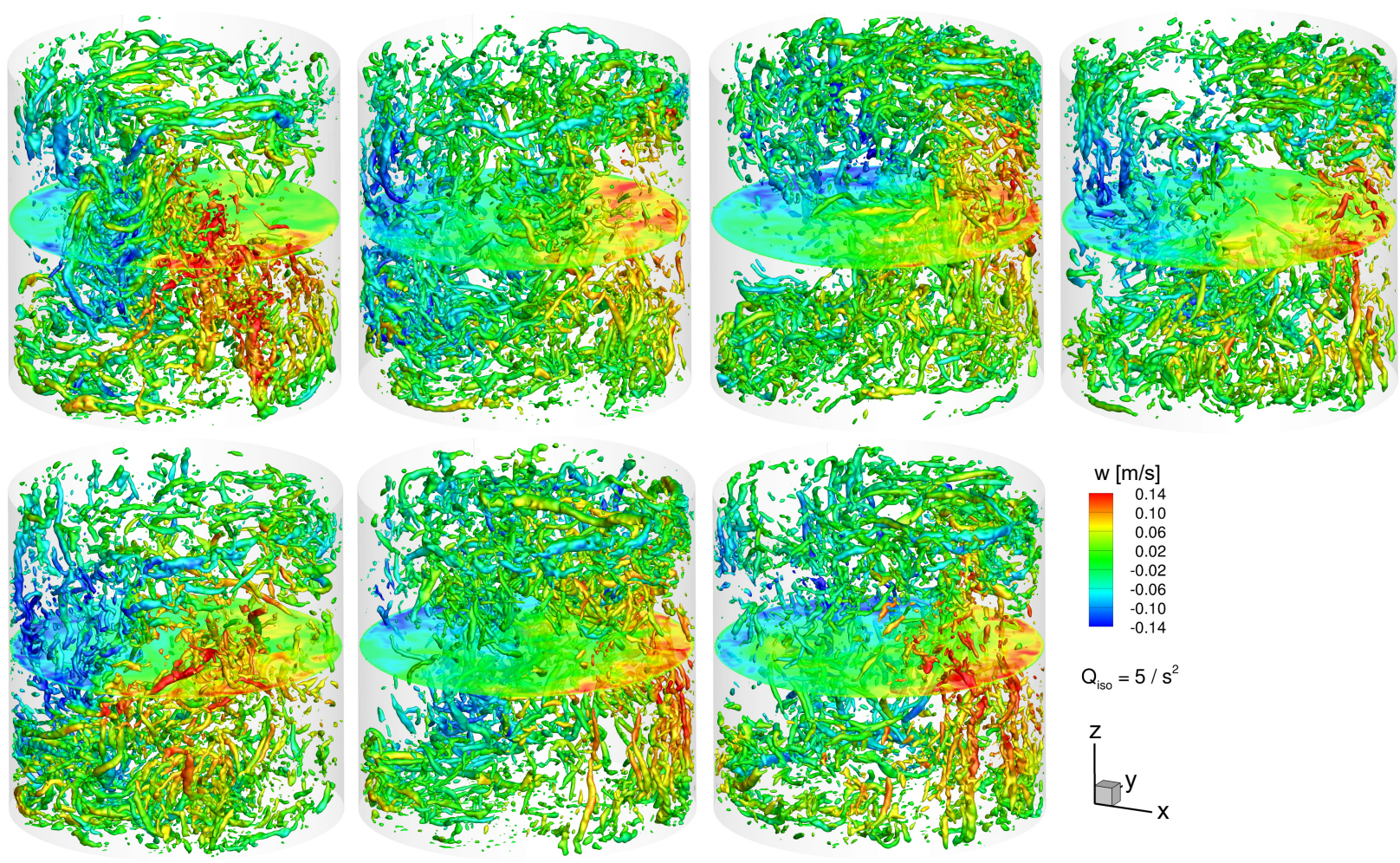

Figure 5: Visualization of instantaneous velocity fields during 'run 09'. Depicted are the iso-surfaces of the Q-criterion and the vertical velocity (color). The first five images are sampled every $10 \mathrm{~s}$ in time.

As the dense particle data impedes visualization of the turbulent structures, regularized data from FlowFit was used in the current section. Hereto, the flow is visualized by iso-surfaces of the Q-criterion (Haller 2005 ) in Figure 5, where the grid spacing of the regularized data amounts to $3 \cdot \eta_{\mathrm{K}}$ at a number of 0.06 tracer particles per cell (PPC). As different instances in time are depicted, the data shall give an impression of the dynamics of the turbulent flow structures. To give insight into an extensive range of turbulent structures with wide size and shape distribution the Q-value for the iso-surfaces was set to $5 / \mathrm{s}^{2}$. In each of the snapshots, the LSC can be spotted in terms of fluid rising in one part of the sample and moving down in another part. Herewith, the axis of rotation is oriented near the y-axis. However, when trying to identify the morphology of the LSC, one has to state that there is no such thing and the LSC rather decomposes into an agglomerate of smaller turbulent structures with very small order. When looking at the vertical velocity data in the central plane, besides the rotation of the LSC, a sloshing motion can be observed, the LSC being located in the front, in the middle and in the back in the different instances. The fine-dynamics of the flow, however, is hidden in the turbulent fluctuations. Regular structures to be observed are discussed in the following. First, at the position, where the LSC detaches from the heating / cooling plate, the turbulent structures rise up and align into the direction of the mean flow, building arrays of aligned structures, spanning more than $1 / 2$ L eventually. At the opposite side, where the LSC reattaches, a corner roller, oriented parallel to the top / bottom plate and oriented perpendicular to the mean flow forms. The dynamics in the region in between the corner roll and the separation is governed by the interaction with the buoyant structures (thermal plumes) with the turbulent flow and shall be elucidated in the next section. 


\subsection{Thermal Plumes and Turbulent Interactions}

Having measured with an oblique view onto the heating plate, we were content to resolve the fingerprints of thermal line-plumes in our velocity data above both, heating and cooling plate. Necessary preconditions to do so were the high scattering efficiency of the HFSBs and some treating of the heating plate with a surfactant to make the bubbles vanish quickly after occasional settling. Figure 6 illustrates the vertical velocity component in a slice located $2.7 \lambda_{\mathrm{b}}$ above the heating plate. Further, the iso-surfaces of the Q-criterion are depicted in a layer of $55 \mathrm{~mm}$ thickness above the plate. Among the most prominent features to be spotted are line plumes, indicated by locally increased vertical velocity values, which are accompanied by pair wise, elongated vortical structures. The latter are induced by the rising plumes and form the well-known mushroom shape, which evolves when line plumes detach from the top or bottom plate. Further, vortical structures rising perpendicular from the heating plate and extending beyond the layer, in which the Q-iso surfaces are plotted, can be spotted. They are caused by interactions of the aforementioned structures with the turbulent LSC flow. Eventually, such structures organize to larger uprising vortical structures, resembling dust devils, known from atmospheric flows.

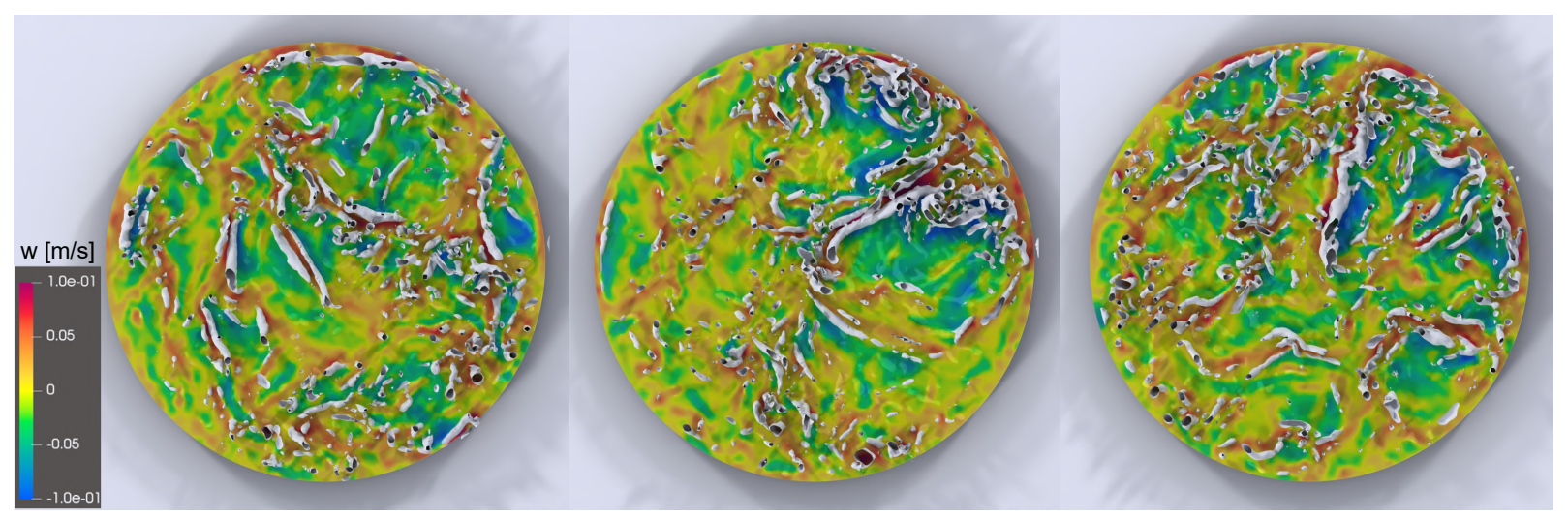

Figure 6: Series with visualization of thermal plumes in instantaneous velocity fields above the heating plate of 'run 30'. Shown are the contours of the vertical velocity in a slice located $23 \mathrm{~mm}$ as well as iso-surfaces of the Q-criterion, $\mathrm{Q}=12 \mathrm{~s}^{-2}$, in a layer of $55 \mathrm{~mm}$ thickness above the heating plate. The interval between the snapshots amounts to one fourth of the LSC turnaround time, i.e. $6.25 \mathrm{~s}$.

\subsection{Lagrangian Statistics}

In order to improve the understanding of the Lagrangian transport processes in our RBC experiment, we evaluated the autocorrelation functions of the different velocity components along the particle tracks, averaging over the 5,000 longest particle tracks of 'run 30'. Herewith, we computed the spatial autocorrelation along the tracks, see Figure 7 (left). The finger print of the LSC can be observed as a strong and periodic correlation signal. The first side maximum is located at $\sim 2.7 \mathrm{~L}$, the mean perimeter of the LSC, corresponding to a LSC turn-around time of $\tau_{\mathrm{LSC}}=25.9 \mathrm{~s}$. It turns out that the residence time of particles in the LSC is quite limited. More precisely, we can conclude from Figure 7, that the motion of the particles, which constitute the LSC, is dephasing in at most two cycles. Obviously, already at the moderate Ra studied in this experiment, the LSC is not to be imagined as a fixed set of fluid particles, which recirculate but rather by a turbulent transport of momentum information, which is exchanged between the particles joining and leaving the roller (very similar findings are reported for superstructures in a TBL by Novara et al. contribution to this ISPIV'21). As a consequence, and a matter of fact, visualization of the LSC at the studied Ra is already quite challenging, because the LSC is mostly of a statistical nature rather than a topological feature of the flow. The conjugated view on these phenomena is given by the power spectral density computed along the tracks, see Figure 7 (middle). The motion of the LSC leads to a prominent peak at about $0.0065(0.037 \mathrm{~Hz})$, which overshadows the inertial subrange scaling at lower frequencies. At higher frequencies, damping of the turbulent structures increases strongly above $\sim \omega_{\eta} / 5$, such that when the cut-off filter of the STB track 
fitting sets on, the structures are already attenuated due to turbulent dissipation by about three orders of magnitude. The onset of the cut-off filter can be clearly spotted and it is located at $\sim 0.6 \omega_{\eta}$. We are thus confident to resolve all relevant turbulent structures up to the dissipation range. However, the aforementioned is valid for the temporal resolution along the tracks. As the particle density decreases with measurement time, the spatial resolution is, of course, decreasing with time. Herewith, one of the assets of Lagrangian measurements as compared to Eulerian is the possibility to provide high track-wise spatial resolution via fast sampling of the particle motion, even at lower seeding densities, see Figure 7 (right), which depicts a PDF of the propagated length in units of the volume averaged Kolmogorov length. It peaks at a value of 2 and shows maximum values of $\sim 5.5$.
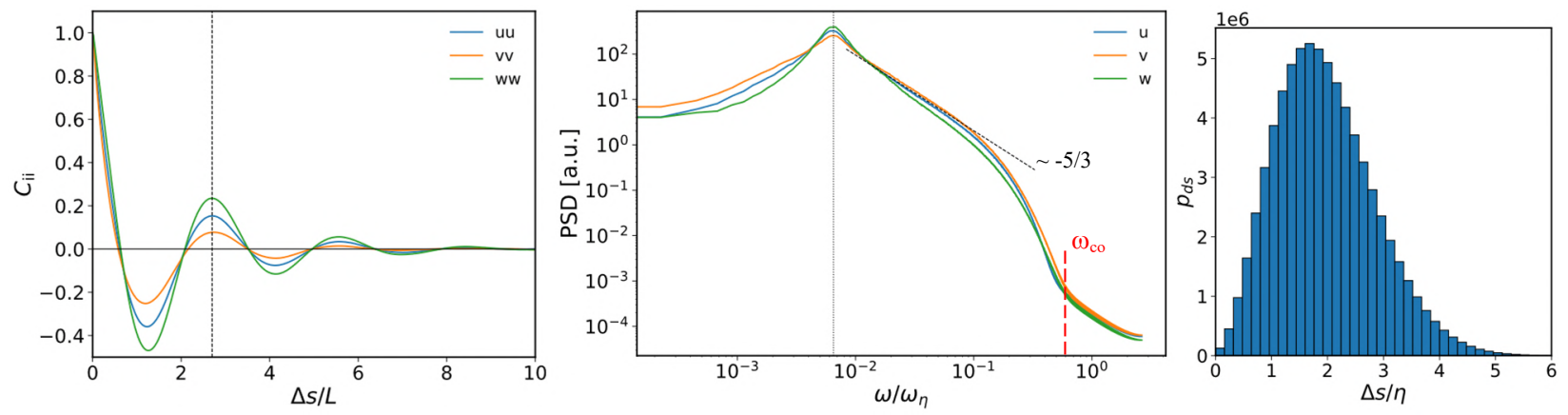

Figure 7: Lagrangian statistics for 'run 30', evaluated over the 5,000 longest tracks: track-wise spatial autocorrelation functions (left) and power spectra (middle). For reference, the inertial subrange scaling is given (dotted line). The frequencies are normalized to the Kolmogorov frequency. PDF of the propagated length in units of the volume averaged Kolmogorov length (right).

\subsection{Dynamics of the Large-Scale Circulation}

To obtain insight into the dynamics and long-term behavior of the LSC, spatial statistics were calculated from the particle data and plotted as time-series in Figure 8 and 10. To clarify, how many tracer particles are required to sample the integral flow data, Figure 8 (left) shows the number of tracked particles together with the total kinetic energy (TKE) of the fluid as a function of time. Obviously, the TKE can be sampled well with only a few thousand particles, which remain at the end of the run, with fluctuations, which are typical of the turbulent RBC system.
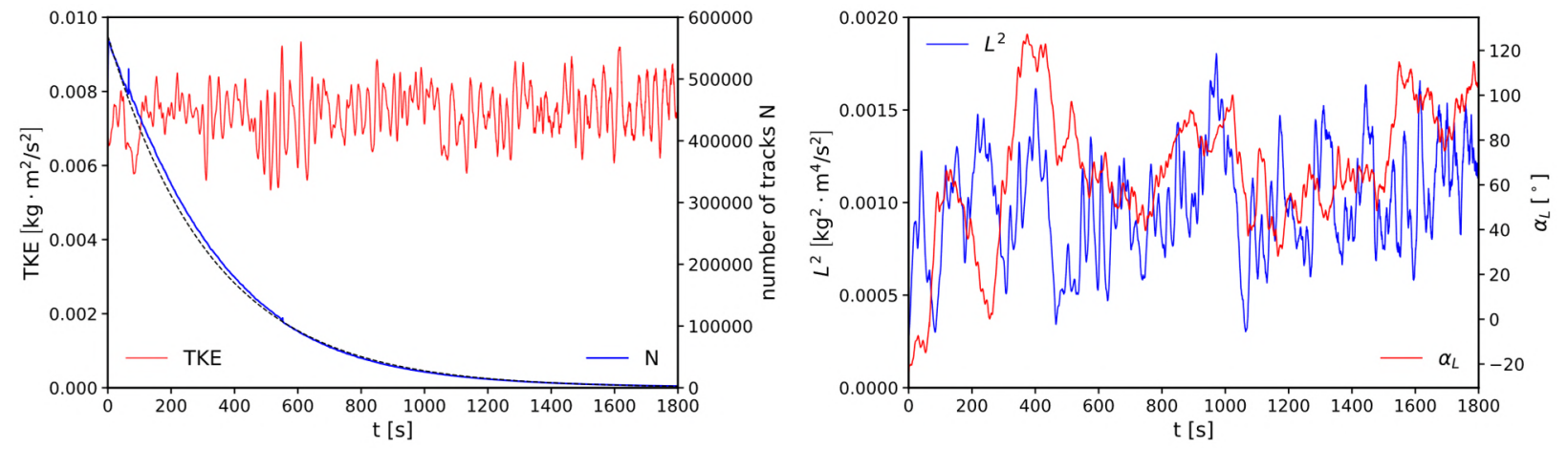

Figure 8: Spatial statistic data as a function of time for 'run 30'. Left: Total kinetic energy (red) and number of tracks (blue). Right: Square of the total angular momentum (blue) and azimuth of the angular momentum (red).

On the right-hand side of Figure 8 the magnitude square and azimuth of the total angular momentum of the fluid is given with respect to the sample center, revealing the dynamic behavior of the LSC. Besides slow meandering of the azimuth ('rotation'), several events involving large angular reorientations, very often in combination with a breakdown of rotational energy, so-called 'spontaneous reorientations' can be spotted. 
In order to further disclose the fine-dynamics of the LSC, the angular momentum was calculated individually for the upper and lower third of the sample. The azimuth between both, $\Delta \alpha^{\mathrm{TB}}$, is plotted in Figure 10. Clearly, the torsional oscillations of the LSC, known from indirect measurements of local wall temperatures (Funfschilling et al. 2008; Zhou et al. 2009), are disclosed by the periodic oscillation of $\Delta \alpha^{\mathrm{TB}}$ with amplitudes up to $80^{\circ}$.

To further unveil the sloshing mode (Zhou et al. 2009) of the LSC from our data as well, we calculated the center of gravity of the projection of the angular momentum to the LSC axis, $\Delta \mathrm{X}_{\alpha}{ }^{\mathrm{COG}}$ (made dimensionless with $\mathrm{L} / 2$ ), see Figure 10. The plot of $\Delta \mathrm{X}_{\alpha}{ }^{\mathrm{COG}}$ versus time reveals the sloshing motion of the LSC, which occurs at the same frequency as the twisting mode. By looking at the autocorrelations of each, $\Delta \mathrm{X}_{\alpha}{ }^{\mathrm{COG}}$ and $\Delta \alpha^{\mathrm{TB}}$, the time constant for these processes, which has been used to normalize the x-axis in Figure 10, could be determined by measuring the first side maximum to be $\tau_{\mathrm{SL}}=28.5 \mathrm{~s}$ (not shown). The latter is higher by about $10 \%$ as compared to the LSC turnaround time determined in the previous section by evaluation of the 5,000 longest tracks. The cross-correlation of $\Delta \mathrm{X}_{\alpha}{ }^{\mathrm{COG}}$ and $\Delta \alpha^{\mathrm{TB}}$ shows, that twisting and sloshing mode are phase locked to each other with a phase shift of $\sim \pi / 4$ in contrast to the phase shift of $\sim \pi / 2$ deduced from wall-temperature measurements (Zhou et al. 2009). In the velocity data, evaluated with the above-mentioned method, the maximal twist occurs already $1 / 8^{\text {th }}$ of a full cycle after the maximal sloshing amplitude.
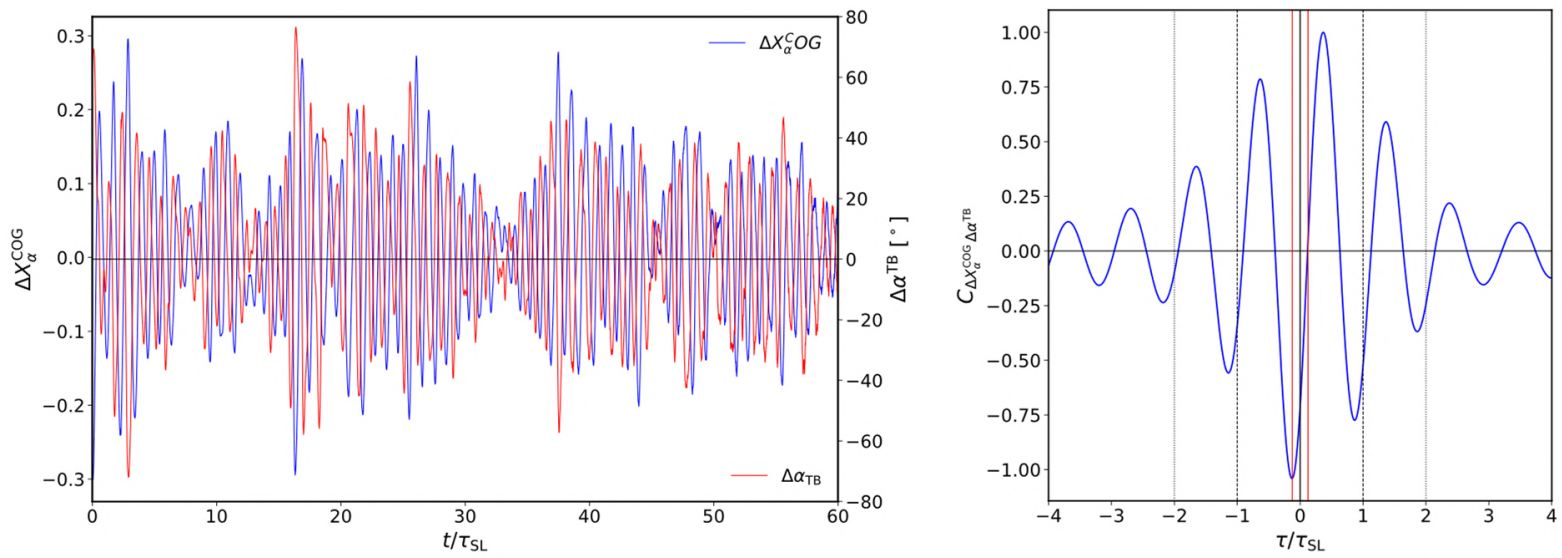

Figure 9: Torsional oscillation and sloshing mode of the LSC during 'run 30': difference of azimuth of the total angular momentum in the upper and the lower third of the sample and center of gravity of the projection of the angular momentum on the LSC axis (left) as well as the cross-correlation of both quantities (right).

\section{Conclusions}

We have demonstrated that by combination of STB / LPT with long-lived HFSB simultaneous acquisition of Lagrangian particle tracks and time-resolved Eulerian velocity fields of turbulent RBC spanning the complete volume of a cylindrical sample becomes possible. With a temporal oversampling factor between 5 and 8 with respect to the Kolmogorov frequency and measurement runs spanning up to 30 minutes, we were able to capture in one measurement short-term dynamics and long-time evolution of the turbulent RBC system. With initial inter-particle distances between 6 and 8 Kolmogorov lengths, spatial resolutions in the assimilated vector fields of a few Kolmogorov lengths could be derived using 'FlowFit'.

The generated data sets allow for direct observation of the interaction between large- and small-scale turbulent structures by visualizations using the Q-criterion. Besides highly resolved views on the LSC, we have been able to detect the fingerprints of thermal plumes rising from the thermal boundary layers in the velocity fields just above the heating and cooling plates. With runs spanning more than 1,000 free-fall times, our data allows spatially and temporally resolved studies of the short and long-term dynamics of the LSC including torsional oscillations, sloshing modes, rotation and spontaneous reorientations. Analysis of the determined time-constants and phase-relations in the light of the results from principal component analysis 
will be among the next steps of our study. The long-lived HFSBs developed in the course of the project with mean lifetimes of more than 5.5 minutes are an important technology for current and future studies of indoor air flow and thermal convection of gaseous fluids (see also paper 184 of this ISPIV'21 by Schröder et al.).

\section{Acknowledgements}

Support with hard- and software for illumination and image acquisition by LaVision GmbH is gratefully acknowledged. We thank J. Lemarechal, T. Kleindienst and C. Fuchs for their contributions to the RBC sample and J. Agocs for his support during set-up of the optical system. J.B. acknowledges several constructive conversations with S. Weiss. We thank the Max-Planck-Institute DS for provision of the refrigerated bath circulator. This work was supported by the Deutsche Forschungsgemeinschaft (DFG) through Grant No. SCHR 1165/5-1 as part of the Priority Programme on Turbulent Superstructures (DFG SPP 1881).

\section{References}

Bodenschatz E, Pesch W, Ahlers G (2000) Recent developments in Rayleigh-Bénard convection. Annu Rev Fluid Mech 32:709-778

Bosbach J, Kühn M, Wagner C (2009) Large scale particle image velocimetry with helium filled soap bubbles. Exp Fluids 46:539-547. https://doi.org/10.1007/s00348-008-0579-0

Brown E, Ahlers G (2006a) Rotations and cessations of the large-scale circulation in turbulent RayleighBénard convection. J Fluid Mech 568:351. https://doi.org/10.1017/S0022112006002540

Brown E, Ahlers G (2006b) Effect of the Earth's Coriolis force on the large-scale circulation of turbulent Rayleigh-Bénard convection. Phys Fluids 18:125108. https://doi.org/10.1063/1.2402875

Chillà F, Schumacher J (2012) New perspectives in turbulent Rayleigh-Bénard convection. Eur Phys J E 35:58. https://doi.org/10.1140/epje/i2012-12058-1

Funfschilling D, Brown E, Ahlers G (2008) Torsional oscillations of the large-scale circulation in turbulent Rayleigh-Bénard convection. J Fluid Mech 607:119-139. https://doi.org/10.1017/S0022112008001882

Gesemann S, Huhn F, Schanz D, Schröder A (2016) From Noisy Particle Tracks to Velocity, Acceleration and Pressure Fields using B-splines and Penalties. In: 18th International Symposium on the Application of Laser and Imaging Techniques to Fluid Mechanics. Lisbon, Portugal, July 4-7

Gibeau B, Gingras D, Ghaemi S (2020) Evaluation of a full-scale helium-filled soap bubble generator. Exp Fluids 61:28. https://doi.org/10.1007/s00348-019-2853-8

Godbersen P, Bosbach J, Schanz D, Schröder A (2020) Video: The beauty of turbulent convection: a particle tracking endeavor. In: 73th Annual Meeting of the APS Division of Fluid Dynamics Gallery of Fluid Motion. American Physical Society, Chicago, IL

Haller G (2005) An objective definition of a vortex. J Fluid Mech 525:1-26. https://doi.org/10.1017/S0022112004002526

Huhn F, Schanz D, Gesemann S, et al (2017) Large-scale volumetric flow measurement in a pure thermal plume by dense tracking of helium-filled soap bubbles. Exp Fluids 58:116. https://doi.org/10.1007/s00348-017-2390-2 
Kühn M, Bosbach J, Wagner C (2009) Experimental parametric study of forced and mixed convection in a passenger aircraft cabin mock-up. Build Environ 44:961-970.

https://doi.org/10.1016/j.buildenv.2008.06.020

Kühn M, Ehrenfried K, Bosbach J, Wagner C (2012) Large-scale tomographic PIV in forced and mixed convection using a parallel SMART version. Exp Fluids 53:91-103.

https://doi.org/10.1007/s00348-012-1301-9

Liot O, Salort J, Seychelles F, et al (2017) Lagrangian Measurements in Turbulent Thermal Convection: About the Inhomogeneity of the Velocity and Temperature Fields. Procedia IUTAM 20:112-119. https://doi.org/10.1016/j.piutam.2017.03.016

Paolillo G, Astarita T, Greco CS, Cardone G (2018) Three-dimensional velocity measurements of Rayleigh-Bénard convection in a cylinder. 18th International Symposium on Flow Visualization, Zurich, Switherland, June 26-29. https://doi.org/10.3929/ethz-b-000279194

Scarano F, Ghaemi S, Caridi GCA, et al (2015) On the use of helium-filled soap bubbles for large-scale tomographic PIV in wind tunnel experiments. Exp Fluids 56:42. https://doi.org/10.1007/s00348015-1909-7

Schanz D, Gesemann S, Schröder A, et al (2013) Non-uniform optical transfer functions in particle imaging: calibration and application to tomographic reconstruction. Meas Sci Technol 24:024009. https://doi.org/10.1088/0957-0233/24/2/024009

Schanz D, Gesemann S, Schröder A (2016) Shake-The-Box: Lagrangian particle tracking at high particle image densities. Exp Fluids 57:70. https://doi.org/10.1007/s00348-016-2157-1

Schanz D, Novara M, Geisler R, et al (2019) Large-scale volumetric characterization of a turbulent boundary layer flow. 13th International Symposium on Particle Image Velocimetry. Munich, Germany, July 22-24

Schiepel D, Herzog S, Wagner C (2018) Experimental Study of Turbulent Rayleigh-Bénard Convection Using Large-Scale Tomo-PIV and High-Density PTV. In: Deville MO, Couaillier V, Estivalezes J-L, et al. (eds) Turbulence and Interactions. Springer International Publishing, Cham, pp 225-231

Spoelstra A, de Martino Norante L, Terra W, et al (2019) On-site cycling drag analysis with the Ring of Fire. Exp Fluids 60:90. https://doi.org/10.1007/s00348-019-2737-y

Sugiyama K, Calzavarini E, Grossmann S, Lohse D (2007) Non-Oberbeck-Boussinesq effects in twodimensional Rayleigh-Bénard convection in glycerol. Europhys Lett EPL 80:34002. https://doi.org/10.1209/0295-5075/80/34002

Wieneke B (2008) Volume self-calibration for 3D particle image velocimetry. Exp Fluids 45:549-556. https://doi.org/10.1007/s00348-008-0521-5

Wieneke B (2013) Iterative reconstruction of volumetric particle distribution. Meas Sci Technol 24:024008. https://doi.org/10.1088/0957-0233/24/2/024008

Zhou Q, Xi H-D, Zhou S-Q, et al (2009) Oscillations of the large-scale circulation in turbulent RayleighBénard convection: the sloshing mode and its relationship with the torsional mode. J Fluid Mech 630:367-390. https://doi.org/10.1017/S0022112009006764 